\title{
LEVELLING OF HEATING AND VEHICLE DEMAND IN DISTRIBUTION NETWORKS USING RANDOMISED DEVICE CONTROL
}

\author{
Peter BOAIT \\ De Montfort University - UK \\ p.boait@dmu.ac.uk
}

\author{
Babak ARDESTANI \\ De Montfort University - UK \\ bmahdavi@dmu.ac.uk
}

\author{
Richard SNAPE \\ De Montfort University - UK \\ jsnape@dmu.ac.uk
}

\begin{abstract}
Rising demand from electrical heating and vehicles will drive major distribution network reinforcement costs unless 24-hour demand profiles can be levelled. We propose a demand response scheme in which the electricity supplier provides a signal to a "smart home" control unit that manages the consumer's appliances using a novel approach for reconciliation of the consumer's needs and desires with the incentives supplied by the signal. The control unit allocates demand randomly in timeslots that are acceptable to the consumer but with a probability biased in accordance with the signal provided by the supplier. This behaviour ensures that demand response is predictable and stable and allows demand to be shaped in a way that can satisfy distribution network constraints.
\end{abstract}

\section{INTRODUCTION}

Authors at a previous CIRED conference have shown that rising demand from electrical heating and vehicles will drive major distribution network reinforcement costs unless 24-hour demand profiles can be levelled [1] - i.e. the ratio of peak demand to average demand needs to be as close to 1 as possible so that the capacity of the network is fully exploited. This involves signalling to consumers to indicate when electricity use should be constrained and when capacity is available. However simply signalling a time dependent price does not always achieve the required demand response and can result in unstable system behaviour. This paper describes a method for reliable levelling of domestic demand, while retaining flexibility for the consumer, using controlled randomisation of the response to signalling.

\section{BACKGROUND}

Domestic demand response schemes typically consist of some combination of electricity tariffs which incentivise response, a signal to the consumer indicating when the response should take place, and agency in the form of manual or automatic control of electricity use that executes the response. An illustrative example is the UK "Economy 7" scheme which offers a discounted tariff for 7 hours overnight and provides a signal embedded in a $198 \mathrm{kHz}$ radio broadcast that indicates the start of the 7 hour interval and causes electric heating appliances with thermal storage to switch on. Also consumers with this tariff often set their own time clocks such that water heating or wet appliance operation is performed during the discounted period.

Research on the effectiveness of smart meters and dynamic pricing of electricity indicates that time-of-use pricing can consistently reduce peak consumption. A range of US trials evaluated by Faruqui and Sergici [2] show reductions of between $2 \%-6 \%$ by user agency, but if "enabling technology" performs the response automatically it results in much higher peak reductions in the range $21 \%-32 \%$.

These results imply that the main burden of demand response should be undertaken by some form of automatic control system which responds flexibly to a price-based signal allowing a wide range of appliances to be brought under control at the consumer's discretion. This conclusion has motivated numerous recent studies of possible schemes by which domestic appliances and charging of electric vehicles might respond automatically to a price signal e.g. [3],[4]. A common feature of all these investigations is that the control unit executing the response has an objective function to minimise cost for the consumer while satisfying the various constraints they may have set such as the latest time that the dishwasher must run. This allows the modelling to show that the consumer would save money. However, cost minimisation as an objective function has a strong tendency to cause demand peaks at times when the cost signalled is at a minimum. This can cause the peak-toaverage ratio (PAR) of the resulting aggregate demand to exceed that of the baseline (i.e. demand with no feedback signal) and hence a key objective of demand response, to reduce PAR, is not achieved.

\section{PROPOSED DEMAND RESPONSE SCHEME}

\section{The home control unit}

The objective function of the proposed domestic automatic demand control unit is to distribute the electrical demand of its controlled appliances over the 48 half hour timeslots in the day (numbered $i=1: 48$ ) in inverse proportion to the 48 valued signal $S$ provided by the electricity supplier at the start of each day. The signal is framed by the supplier and interpreted by the control unit as if it is a cost, but the response to the signal in each timeslot is arranged to be proportional to the deviation from the mean, as far as is possible within the consumer's constraints on individual appliances. 
This proportionality is achieved by displacing demand within the time window acceptable to the consumer to alternative timeslots with a probability proportional to the amplitude of the signal in each timeslot of the window. Because the probabilities over the window must add up to 1 , this means that for any $S$ with more than one non-zero timeslot in the window some redistribution of demand will take place giving this method an inherent tendency to reduce peaks in demand. Taking the example of a wet appliance that the consumer has specified must run sometime in the next $n$ timeslots with a duration of $k$ timeslots, the controller will invert $S$ to create an "attractiveness" vector $\boldsymbol{A}_{\boldsymbol{w}}$ with values given by $A_{w i}=\left(S_{\max }\right.$ $-S_{i}$ ) where $S_{\max }$ is the largest value of $\boldsymbol{S}$. The controller then uses a random number generator to select one of the $n$ $k+1$ possible timeslots in which the appliance can start, with a probability $P_{w i}$ given by:

$$
P_{w i}=\sum_{j=i}^{j=i+k-1} A_{w j} /\left(\sum_{q=1}^{q=n-k+1} \underset{r=q}{r=q+k-1}\left(\sum_{w r} A_{w r}\right)\right)
$$

The effect is that over a population of consumers choosing a similar time window demand will be allocated in proportion to the possible alternative averages of $A_{w}$ over $k$ timeslots. This approach is applied to heat pumps by introducing a gap in heating demand when $S_{i}>0$ for one or two timeslots such that the fall in room temperature is less than $0.5^{\circ} \mathrm{C}$. The heat pump is regulated by the control unit using the methods described in [5] making use of the thermal mass of the building such that the gap either provides energy saving or recovery of the heat input is spread over other timeslots so that there is no surge in demand after the gap. The probability $P_{h i}$ for a single timeslot gap out of the set of $p$ timeslots for which $S_{i}>0$ is given by:

$$
P_{h i}=S_{i} / \sum_{j=1}^{j=p} S_{j}
$$

Charging of electric vehicles from the domestic supply follows the same concept. The home control unit learns the usage pattern of a vehicle under control so that when it is reconnected after use the control unit has a prediction of both the amount of charge $Q_{t o t}$ required to restore the battery to full capacity and the next time at which the vehicle will be needed. It then allocates this expected charge $Q_{t o t}$ over the $r$ timeslots available in proportion to the attractiveness $A_{v i}=\left(S_{\max }-S_{i}\right)$ of each timeslot. The charge $Q_{i}$ in each timeslot is given by:

$$
Q_{i}=Q_{t o t} A_{v i} / \sum_{j=1}^{j=r} A_{v j}
$$

\section{Supplier's model of demand response}

Given a customer base of consumers equipped with control units as described above, it can be seen that the total demand in each timeslot seen by the supplier will vary proportionately to $S_{i}$ but with different constants of proportionality $k_{i}$ for each timeslot and for positive and negative values of $S_{i}$. These variations in proportionality arise from the differing constraints applied to each class of appliance across the consumer population. However, if the supplier can determine the 96 linear functions predicting the response to $S$ it can construct $S$ to achieve a range of objectives which are explored in the next section. It is assumed that the supplier is able to predict the 48 value baseline demand vector $\boldsymbol{B}$ that is expected for any day, using established records and techniques such as neural network forecasting. Then by transmitting different experimental constructions of $\boldsymbol{S}$ over a suitable period and recording the resulting difference $\partial B_{i}$ in demand in each timeslot relative to the known baseline, the supplier can construct a sufficient number of equations of the form:

$$
\partial B_{i}=S_{i} k_{i} B_{i}+c_{i}
$$

to solve for the 96 values of $k_{i}$ and $c_{i}$. The demand $\boldsymbol{D}$ arising from any $S$ can then be predicted using:

$$
D_{i}=B_{i}\left(1+S_{i} k_{i}\right)+c_{i}
$$

where $k_{i}$ and $c_{i}$ are taken from the $\boldsymbol{k}$ and $\boldsymbol{c}$ vectors appropriate to the sign of $S_{i}$. Once in operation this model can be updated continuously from metering records. In practice the supplier may wish to transmit a null $S$ to a proportion of consumers, either to sense the current baseline demand, or to achieve a particular shape of aggregate demand.

\section{SIMULATION OF SCHEME}

\section{Domestic demand model}

To evaluate the properties of this approach for shaping domestic demand, a model has been constructed which simulates the electricity consumption of a population of households equipped with a control unit and appliances that respond to a signal as specified in the previous section. In order to obtain realistic demands for space and water heating, the occupancy and building thermal properties of these households were assigned to correspond with UK national statistics [6], [7]. For each class of appliance, a default daily demand profile was created giving the default probability that the appliance was operating in any given timeslot. The demand when operating was calibrated to match either the average demand for this class of appliance from national statistics, or the actual thermal load of the simulated household as determined by its thermal properties. The electrical load $W_{s}$ for space heating is given in $\mathrm{kW}$ by:

$$
W_{s}=L\left(T_{r}-T_{e}\right) / c
$$

where $c$ is the coefficient of performance $(\mathrm{CoP})$ of the heat pump, $L$ is the loss rate for the dwelling, $T_{e}$ is the external ambient temperature, and $T_{r}$ is the reference temperature $\left(15.5^{\circ} \mathrm{C}\right)$ which space heating must achieve, the remaining 
heat input for higher room temperatures being provided by appliance use, occupancy, and solar gain. The change in room temperature $T_{r o}$ when heating is ceased for one or two timeslots is calculated using a single node model of the building characteristics:

$$
\left(T_{r o}-T_{e}\right)=\tau d T_{r o} / d t, \quad \tau=C / L
$$

where $C$ is the thermal mass and $L$ the loss rate. CoPs of 2.5 and 2.2 for space heating and water heating respectively are employed, based on Energy Saving Trust findings for UK installations [8].

For simulation of electric vehicle (EV) use a single EV was assumed in $25 \%$ of homes. $50 \%$ of EVs were assumed to have a performance equivalent to the Nissan Leaf, and the remaining 50\% were assumed equivalent to the Vauxhall Ampera. Baseline electricity use was determined by the profile of arrival times at home (shown in Figure 1) and trip distances derived from survey data [9] resulting in a distribution of charge requirements that are satisfied at a $3 \mathrm{~kW}$ rate (constrained by the UK 13 Amp household socket).

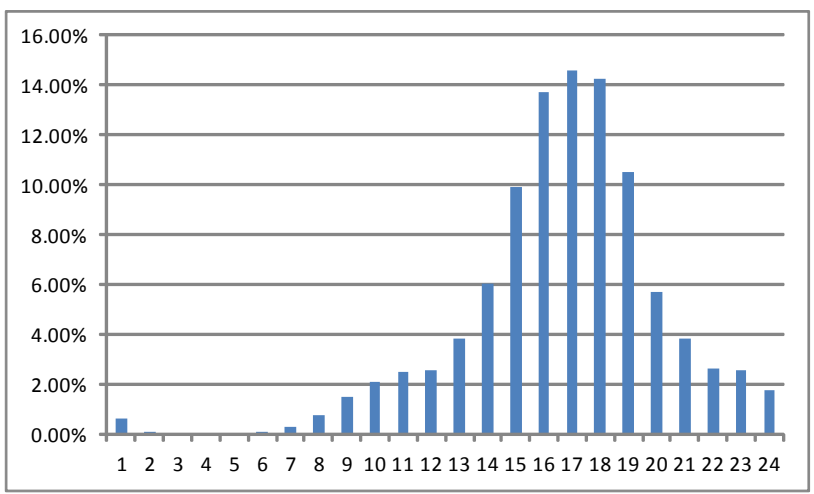

Figure 1. Distribution of electric vehicle return times

\section{$\underline{\text { Results }}$}

The results presented below share a common scenario of 1000 households with space and water heating by heat pumps, and appliance and electrical vehicle use as specified above. A winter day with overnight temperatures falling to $-1.4{ }^{\circ} \mathrm{C}$ is simulated as this is likely to represent a worstcase load on local distribution networks. A typical resulting baseline demand profile from these households is shown in Figure 3 including the contributions from the various appliance types - the miscellaneous type covers all those appliances such as entertainment devices that are only amenable to manual control.

The demand-flattening potential is fully exploited when the supplier uses the demand response model to generate a signal optimised to induce a flat response, with an objective function using equation (5):

Minimise: $D_{\max } / \bar{D}$ where $D_{i}=B_{i}\left(1+S_{i} k_{i}\right)+c_{i}$
The simulation employs the "active set" method to solve this non linear optimisation problem for $S$. The response of the consumer population with a baseline demand as indicated in Figure 2 to a signal $S$ determined in this way is shown Figure 3, which has a PAR of 1.09. This may be compared with the baseline PAR of 1.4. The residual variations in demand arise from the imperfections in the demand model held by the supplier and the various constraints on electricity use applied by consumers.

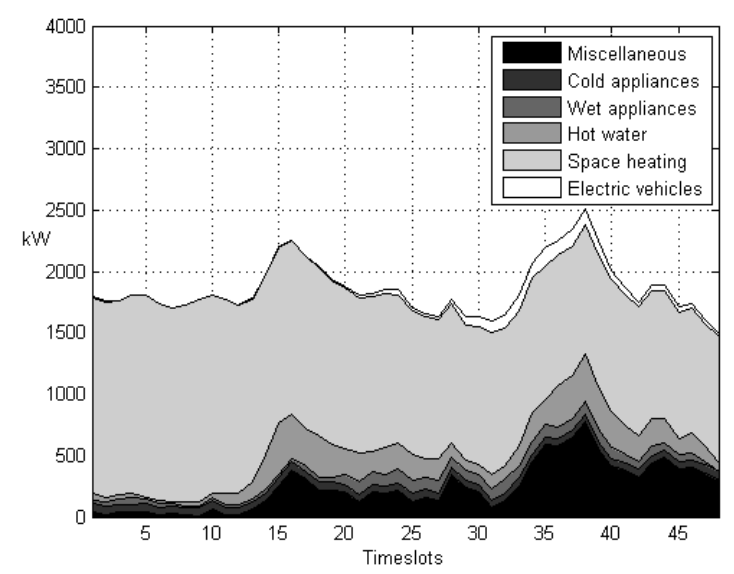

Figure 2. Baseline winter demand from consumer model1000 households

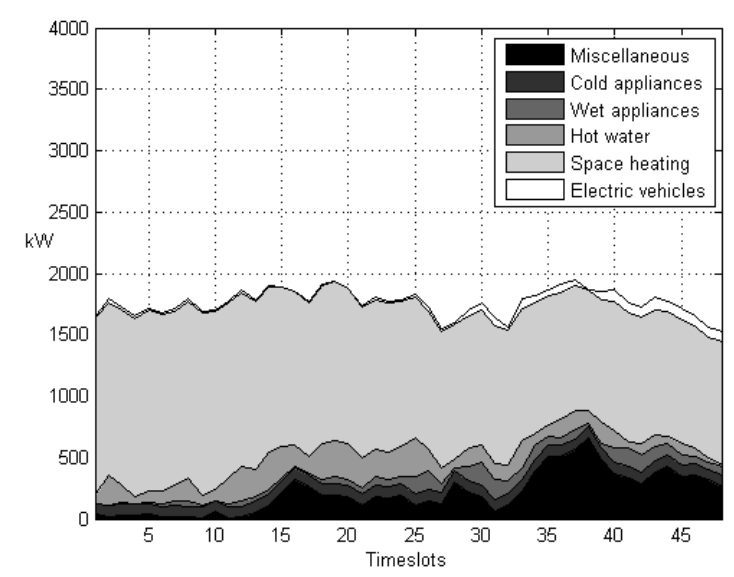

Figure 3. Demand response to a signal optimised for flattening

Where distribution network capacity permits, the supplier can employ a suitably optimised $\boldsymbol{S}$ to obtain any desired shape of demand within the flexibility limits determined by consumer preferences and needs. In Figure 4 it is envisaged that the supplier has access to a surge of wind generation overnight which is expected to reduce later in the day. A signal optimised to a target profile lifting demand between 00:00 and 07:30 attracts an additional $3300 \mathrm{kWh}$ of demand into the early hours amounting to $7.7 \%$ of the demand for the day. Had the wind surge been expected later in the day the supplier could simply have sent a null signal allowing the baseline demand peak to occur. 


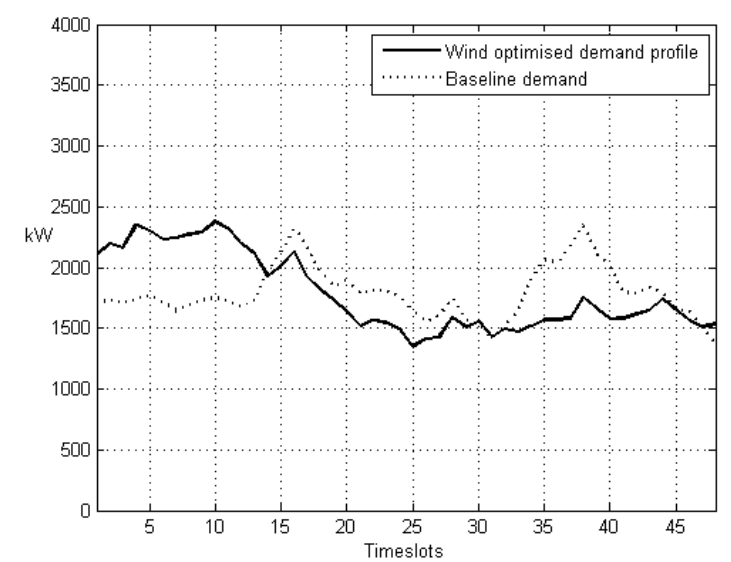

Figure 4. Demand optimised for overnight wind generation availability

\section{CONCLUSION}

The simulation results show that this method for inducing demand response is capable of both reducing demand peaks and moving demand in time to coincide with renewable electricity generation. It also has several other useful properties:

1. It is completely scalable in that the signal can be directed at a few households or millions.

2. For many desirable demand profiles, such as the flattened profile shown in Figure 3, the amount of electricity consumed is reduced through user action to switch off loads in response to the signal being interpreted and displayed as a higher price.

3. A single home control device can manage all appliance types and electric vehicles using a common underlying concept. There is no need for a specialised electric vehicle charging controller the concept is readily adaptable to "vehicle to grid" schemes by allowing limited and proportionate export of energy from the vehicle battery in timeslots where $\boldsymbol{S}$ is positive. It is similarly applicable to despatch of micro CHP units. This simplification and commonality will aid user understanding and acceptance.

4. Because the PAR of typical demand profiles is always reduced, the gain in the consumer response element of the demand response feedback loop is less than 1 , so stability is enhanced in wholesale market pricing and total demand.

A critical issue this scheme may help to resolve is the relationship between suppliers and distribution network operators (DNOs). Both can gain from the ability to shape demand but have different objectives. Use of this method in a suitable regulatory context to reconcile these conflicting interests is presented in Session 6 (paper 0289).

\section{Acknowledgement}

The authors would like to thank the Engineering and Physical Sciences Research Council (EPSRC) for providing the financial support for this study as part of the CASCADE project (EP/GO59969/1).

\section{REFERENCES}

[1] C. Gan, M. Aunedi, V. Stanojevic, G. Strbac, D. Openshaw, 2011, "Investigation of the impact of electrifying transport and heat sectors on the UK distribution networks", Proc 21st Int. Conf. on Electricity Distribution (CIRED), paper 0710

[2] A. Faruqui, S. Sergici, 2010, "Household response to dynamic pricing of electricity: a survey of 15 experiments", J Regul Econ, vol. 38, 193-225.

[3] A. Roscoe, G. Ault, 2010, "Supporting high penetrations of renewable generation via implementation of real-time electricity pricing and demand response", IET Renew. Power Gener., vol 4(4), 369-382.

[4] D. Papadaskalopoulos, G. Strbac, 2011, "Decentralised agent-based participation of load appliances in electricity pool markets", Proc 21st Int. Conf. on Electricity Distribution (CIRED), paper 1049

[5] P. Boait, D. Fan, A. Stafford, 2011, "Performance and control of domestic ground-source heat pumps in retrofit installations", Energy and Buildings, vol. 43, 1968-1976.

[6] DECC, 2010,"Energy consumption in the UK domestic data tables", http://www.decc.gov.uk/en/content/cms/statistics/pu blications/ecuk/ecuk.aspx

[7] DCLG, 2011, "English Housing Survey Headline Report 2010-11", http://www.communities.gov.uk/publications/corpor ate/statistics/ehs201011headlinereport

[8] Energy Savings Trust, 2010, "Getting warmer: a field trial of heat pumps", http://www.energysavingtrust.org.uk/Generate-yourown-energy/Heat-pump-field-trial/Getting-warmera-field-trial-of-heat-pumps-PDF

[9] DfT, 2010, "National Travel Survey", http://www.dft.gov.uk/statistics/releases/nationaltravel-survey-2010 